Published in:

Computers and Industrial Engineering, Vol. 26, No. 3, pp. 609-618, 1994.

\title{
A COMPUTATIONAL EVALUATION OF THE ORIGINAL AND REVISED ANALYTIC HIERARCHY PROCESS
}

\author{
EVANGELOS TRIANTAPHYLLOU ${ }^{1}$ and STUART H. MANN ${ }^{2}$
}

1 A ssistant Professor, Department of Industrial and M anufacturing Systems Engineering, Louisiana State University, 3134C CEBA Building, Baton Rouge, LA 70803-6409, U.S.A.

E-mail: ietrian@Isuvm.sncc.Isu.edu

Web: http://www.imse.Isu.edu/vangelis

2 Professor of Operations Research and Director of SHRIM, The Pennsylvania State University, University Park, PA 16802, U.S.A.

\begin{abstract}
The A nalytic Hierarchy Process (AHP) and its variants have long been used in numerous scientific and engineering applications. The present paper demonstrates that the original AHP and one of its variants have the potential to reach the wrong conclusion under certain circumstances. This paper examines the effectiveness of these two methods under the assumption that in reality the pairwise comparisons, which are used in these methods, take on continuous values. This assumption is made in order to capture the majority of the real world cases. The computational results in this paper demonstrate that when the above assumption is made, the A HP and the revised A HP might yield a different ranking of the alternatives than the ranking that would result if the actual relative importances were known. The same results also reveal a dramatic increase in the probability that an incorrect ranking occurs as the number of alternatives involved increases.
\end{abstract}

\section{All correspondence should be addressed to the first author.}




\section{INTRODUCTION}

The A nalytic Hierarchy Process (A HP) is best illustrated by Saaty in [1], [2], and [3]. The AH P has attracted the interest of many researchers mainly due to the nice mathematical properties of the method and the fact that the required input data are rather easy to obtain (see, for example, [4], [5], [6], [7], [8], [9], [11], and [12]). The AHP is a decision support tool which can be used in solving complex decision problems. It uses a multi-level hierarchical structure of objectives, criteria, subcriteria, and alternatives. The pertinent data are derived by using a set of pairwise comparisons. These comparisons are used to obtain the weights of importance of the decision criteria, and the relative performance measures of the alternatives in terms of each individual decision criterion. If the comparisons are not perfectly consistent, then it provides a mechanism for improving consistency.

The typical problem examined by the A HP consists of a set of alternatives and a set of decision criteria. Since this problem is very common in many engineering applications, A HP has been a very popular decision tool. A nother reason which contributed to the wide use of AHP in engineering applications, is the development of the Expert Choice software [13]. Furthermore, many other computer packages have been developed and are based on the principles of the AHP. Some of the engineering applications of the AHP include its use in integrated manufacturing [14], and [15], in the evaluation of technology investment decisions (i.e., [16], [17], [18], and [19]), in flexible manufacturing systems [20], layout design [21], and also in other decision problems (see, for instance, [22], and [23]).

The revised AHP is a variant of the original AHP and was proposed by Belton and Gear [24]. They observed that the A HP may reverse the ranking of the alternatives when an alternative identical to one of the already existing alternatives is introduced. In order to overcome this deficiency, Belton and Gear proposed that each column of the A HP decision matrix to be divided by the maximum entry of that column.

The fact that rank reversal also occurs with near copies are considered, has also been studied by 
Dyer and Ravinder [25] and Dyer and Wendell [26]. In [27] and [28] Saaty provided some axioms and guidelines on how close a near copy can be to an original alternative without causing a rank reversal. $\mathrm{He}$ suggested that the decision maker has to eliminate alternatives from consideration that score within 10 percent of another alternative. This recommendation was later sharply criticized by Dyer [29].

In [29] and [30] Dyer demonstrated that the original A HP may result in arbitrary rankings, even when no identical alternatives are considered. He also admits that in some cases the AHP may yield results which may be highly correlated with the true performances of a consistent decision maker, and even provide an accurate ranking of alternatives in other special cases, however, he asserts that in general this cannot be guaranteed a priori.

He attributed this problem to the principle of hierarchic composition, which is one of the main assumptions in the AHP. This principle assumes that the weights of the criteria do not depend on the al ternatives under consideration. As an alternative approach, Dyer suggested that A H P be combined with utility theory. That paper by Dyer stimulated some responses by Saaty [31] and Harker and V argas [32]. They expressed many concerns whether traditional utility theory can be combined with the A HP.

As W inkler [33] remarked in a related technical note, utility theory is not a panacea. He cautions the reader to be careful in accepting alternative theories. Clearly, these problems are still controversial in decision theory. Some additional discussions on these truly important issues are due to Bell and Farquhar [34], Hogarth and Reder [35], Weber and Camerer [36], M unier [37], Fishburn and L aV alle [38], and Sarin [39].

The A HP and the revised A HP, along with two other decision-making methods, were compared by Triantaphyllou and $\mathrm{M}$ ann in [8] by applying two evaluative criteria. The first evaluative criterion had to do with the premise that a decision method which is accurate in multi-dimensional problems should also be accurate in single-dimensional problems. There is no reason for an accurate multi-dimensional method to fail in giving accurate results in single-dimensional problems, since single-dimensional problems 
are special cases of multi-dimensional ones. The second evaluative criterion considered the premise that an efficient decision-making method should not change the indication of the best alternative when an alternative (not the best) is replaced by another worse alternative (given that the importance of each decision criterion remains unchanged). In that investigation the revised A HP performed significantly better than the original A HP and for that reason the revised A HP is considered in the present investigation.

One fundamental step in the A HP and the revised A HP is the manipulation of a reciprocal matrix with pairwise comparisons. These pairwise comparisons reflect the estimates made by the decision maker of the relative importance of each al ternative in terms of a given decision criterion. In applications of the A HP to real decision-making problems the entries in the above reciprocal matrix are taken from the finite set: $\{1 / 9,1 / 8, \ldots, 1,2, \ldots, 8,9\}$ (which has been suggested by Saaty to be appropriate [3]). This is achieved by using the scale presented in table 1 . That is not to say that a discrete set or even continuous values could not be used, for it is known that even continuous values satisfy the Saaty axioms [27]. H owever, in practice the above discrete set is almost always used. A fter a decision maker forms the entries of all the pertinent reciprocal matrices, an approach based on eigenvalue theory is used to derive the relative weights of each criterion and also of each alternative in terms of each criterion in a decision-making problem. An investigation on the development and performance of alternative scales for quantifying pairwise comparisons can be found in [40].

Triantaphyllou and $M$ ann in [11] tested the eigenvalue approach under a data continuity assumption. According to this assumption the actual (and thus unknown) values of the pairwise comparisons take on continuous values and not necessarily discrete ones. That continuity assumption is made in order to capture the continuous nature of most real life decision-making problems. In that investigation it was found that the eigenvalue approach is likely to yield a ranking of elements that is different than the ranking yielded when actual data were used. 
Table 1. Scale of Relative Importances [3]

\begin{tabular}{|c|c|c|}
\hline $\begin{array}{l}\text { Intensity of } \\
\text { Importance }\end{array}$ & Definition & Explanation \\
\hline 1 & Equal importance & $\begin{array}{l}\text { Two activities contribute } \\
\text { equally to the objective }\end{array}$ \\
\hline 3 & $\begin{array}{l}\text { Weak importance of one } \\
\text { over another }\end{array}$ & $\begin{array}{l}\text { Experience and judgment } \\
\text { slightly favor one } \\
\text { activity over another }\end{array}$ \\
\hline 5 & $\begin{array}{l}\text { Essential or strong } \\
\text { importance }\end{array}$ & $\begin{array}{l}\text { Experience and judgment } \\
\text { strongly favor one } \\
\text { activity over another }\end{array}$ \\
\hline 7 & $\begin{array}{l}\text { Demonstrated } \\
\text { importance }\end{array}$ & $\begin{array}{l}\text { A } n \text { activity is strongly } \\
\text { favored and its dominance } \\
\text { demonstrated in practice }\end{array}$ \\
\hline 9 & Absolute importance & $\begin{array}{l}\text { The evidence favoring one } \\
\text { activity over another is } \\
\text { of the highest possible } \\
\text { order of affirmation }\end{array}$ \\
\hline $2,4,6,8$ & $\begin{array}{l}\text { Intermediate values } \\
\text { between the two } \\
\text { adjacent judgments }\end{array}$ & When compromise is needed \\
\hline $\begin{array}{l}\text { Reciprocals of } \\
\text { above nonzero }\end{array}$ & $\begin{array}{l}\text { If activity i has one } \\
\text { of the above nonzero } \\
\text { numbers assigned to it } \\
\text { when compared with } \\
\text { activity } j \text {, then } j \text { has } \\
\text { the reciprocal value } \\
\text { when compared with i. }\end{array}$ & \\
\hline
\end{tabular}

In the present investigation we follow a similar approach as in [11]. Since a decision-making problem involves a sequence of reciprocal matrices, the testing strategy developed in [11] is repeated a number of times. One time for each one of the reciprocal matrices involved in a given decision problem. Recall that if a problem is defined on $\mathrm{N}$ decision criteria and $\mathrm{M}$ alternatives, then there is one such matrix of order $\mathrm{N} \times \mathrm{N}$ and $\mathrm{N}$ matrices of order $\mathrm{M} \times \mathrm{M}$. 
In [11] it was found that when the eigenvalue method is used to derive relative weights, then it is possible for some items (that is, decision criteria or alternatives) to have the same rank while in reality they are distinct. H owever, the present paper demonstrates that it is possible alternatives which in reality are less important than others to appear to be more important after the AHP or the revised AHP are used. Furthermore, this problem of rank reversals is more severe in the present investigation. Also, the present findings reveal that the rate of the rank reversals increases dramatically as the number of alternatives increases.

In the following sections we briefly review the notion of the Saaty reciprocal, the eigenvalue approach of deriving weights, the AHP method, the revised AHP method, as well as other relevant concepts.

\section{SOME BACKGROUND INFORMATION}

\section{Reciprocal M atrices with Pairwise Comparisons.}

Let $A_{1}, A_{2}, \ldots, A_{n}$ be the members of a fuzzy set. This can be a set of alternatives which the decision maker needs to evaluate in terms of a singe decision criterion. We are interested in evaluating the membership values (i.e., the relative weights) of the above members. Saaty [1], [2], [3] proposes to use a matrix $A$ of rational numbers taken from the finite set: $\{1 / 9,1 / 8, \ldots, 1,2, \ldots, 8,9\}$. Each entry of the above matrix A represents a pairwise judgment. Specifically, the entry $a_{i j}$ denotes the number which estimates the relative membership value of element $A_{i}$ when it is compared with element $A_{j}$. Obviously, $a_{i j}=1 / a_{j i}$ and $a_{i i}=1$. That is, the matrix $A$ is a reciprocal one. Saaty in [3] asserts that the desired relative membership values are the elements of the principle right eigenvector of matrix $A$. More discussion on the problem of how to estimate relative membership values from such matrices can be found in [10].

Saaty estimates the principal right eigenvector of the reciprocal matrices by multiplying the entries 
in each row of matrix $A$ together and then taking the $n$-th root ( $\mathrm{n}$ is the number of the elements in the fuzzy set). Since in the A HP it is desired to have values that add up to 1.00 the previously found vector is normalized by dividing each entry by the sum of the above values. If we want (as is the case in the revised AHP method) the element with the highest value to have membership value equal to 1.00 , then we need to divide the previously found vector by the highest value.

\section{The Analytic Hierarchy Process.}

Part of the analytic hierarchy process [3] deals with the structure of an $\mathrm{MxN}$ decision matrix (where $\mathrm{M}$ is the number of alternatives and $\mathrm{N}$ is the number of criteria). This matrix is constructed using the relative importances of the alternatives in terms of each criterion. The vector $\left(a_{i 1}, a_{i 2}, a_{i 3}, \ldots\right.$, $\left.a_{i N}\right)$, for $i=1,2,3, \ldots, M$, is the principal right eigenvector of an $\mathrm{N} \times \mathrm{N}$ reciprocal matrix. This matrix is formed by the pairwise comparisons which express the relative importance of pairs of al ternatives in terms of the i-th decision criterion.

Some evidence is presented in [3] which supports this technique for eliciting numerical evaluations of qualitative phenomena from experts and decision makers. The above $\mathrm{NxN}$ reciprocal matrices are processed as in the previous section. That is, the $\mathrm{a}_{\mathrm{ij}}$ values are determined by using the eigenvalue method. The entry $a_{i j}$, in the $M x N$ decision matrix, represents the relative value of the alternative $A$ when it is considered in terms of criterion j. In the original A HP the sum $\sum_{i=1}^{N} \mathrm{a}_{\mathrm{ij}}$ is equal to one.

According to AHP the best alternative (in the maximization case) is indicated by the following relationship:

$$
A_{A H P}^{*}=\max \sum_{j=1}^{N} a_{i j} w_{j} \quad \text { for } i=1,2,3, \ldots, M
$$




\section{The Revised Analytic Hierarchy Process.}

Belton and Gear [24] propose a revised version of the original A HP model. They demonstrate that an inconsistency can occur when the AHP is used. A numerical example is presented in [24] which consists of three decision criteria and three alternatives. The indication of the best alternative changes when an alternative identical to one of the nonoptimal alternatives is introduced (thus creating four alternatives). According to the authors the root for that inconsistency is the fact that the relative values for each criterion sum up to one. Instead of having the relative values of the alternatives $A_{1}, A_{2}, A_{3}, \ldots$, $A_{M}$ sum up to 1.00 , they propose to divide each relative value in the columns of the decision matrix by the maximum value of that column.

\section{THE CONCEPTS OF THE RCP AND CDP MATRICES}

The following forward error analysis is based on the assumption that in the real world the membership values in a fuzzy set take on continuous values. This assumption is believed to be a reasonable one since it captures the majority of real world cases. As it was mentioned earlier, these members could be a set of alternatives to be examined in terms of a given decision criterion. In this case the membership function expresses the degree that these alternatives meet the single decision criterion.

Let $\omega_{1}, \omega_{3}, \omega_{3}, \ldots, \omega_{n}$ be the real (and thus unknown) membership values of a fuzzy set with n members. If the decision maker knew the above real values, then he would be able to construct a matrix with the real pairwise comparisons. In this matrix, say matrix $A$, the entries are $\alpha_{i j}=\omega_{i} / \omega_{j}$. This matrix is called the Real Continuous Pairwise matrix, or the $\underline{\mathbf{R C P}}$ matrix. Since in the real world the $\omega_{i}^{\prime}$ 's are unknown, so are the entries $\alpha_{i j}$ of the previous matrix. However, we can assume here that the decision maker instead of an unknown entry $\alpha_{i j}$ is able to determine the closest values taken from the set: $\{1 / 9,1 / 8, \ldots, 1,2, \ldots, 8,9\}$. That is, instead of the real (and thus unknown) value $\alpha_{i j}$ one is able to determine $a_{i j}$ such that: 


$$
\begin{aligned}
& \text { the difference }{ }^{*} \alpha_{i j}-a_{i j} * \text { is minimal, } \\
& \text { and } a_{i j} \in\{1 / 9,1 / 8,1 / 7, \ldots, 1,2, \ldots, 7,8,9\} \text {. }
\end{aligned}
$$

In other words, it is assumed here that one's judgments about the value of the pairwise comparison of the $\mathrm{i}$-th element when it is compared with the $\mathrm{j}$-th one, is so accurate that in real life it is the closest (in absolute value terms) of the values one is supposed to choose from. A pparently, this assumption favors the values of the failure rates derived later in this paper, since in reality it is very unlikely that a decision maker can be that accurate all the time. This observation indicates that even the dramatic high failure rates, which are demonstrated in a later section, are smaller than the actual failure rates which may occur when the A HP or the revised AHP are applied on real life problems.

The matrix with the $a_{i j}$ entries that we assume the decision maker is able to construct has entries from the discrete and finite set: $\{1 / 9,1 / 8, \ldots, 1,2, \ldots, 8,9\}$ (when the original Saaty scale in table 1 is used).

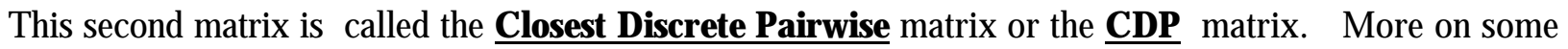
interesting properties of RCP and CDP matrices can be found in [40].

In the numerical example of the following section, it is assumed that the actual relative values in a decision-making problem, which involves 3 alternatives and 4 criteria, are known. The AHP and the revised AHP are applied to these actual data and the relative weight and ranking of each alternative is derived according to each decision-making method.

Next, we use the concepts of the RCP and CDP matrices to derive the discrete data. These discrete data are the data which a decision maker could have if one were able to make the closest approximations to the real (and thus unknown) pairwise comparisons. Again, this is a favorable assumption since there is no certainty that in reality one can achieve these closest approximations for all the pairwise comparisons. The following numerical example reveals that it is possible for the AHP and the revised A HP method to fail to preserve the ranking of the al ternatives when the continuity assumption is utilized and even if the decision maker is as accurate as possible. 


\section{A NUMERICAL EXAMPLE}

Suppose that a decision-making problem with 3 alternatives and 4 criteria has in reality the following matrix as the decision matrix with the actual relative weights:

\begin{tabular}{|c|c|c|c|c|}
\hline \multicolumn{5}{|c|}{ Criterion } \\
\hline & 1 & 2 & 3 & 4 \\
\hline Alt. & (0.1325 & 0.0890 & 0.5251 & $0.2533)$ \\
\hline$A_{1}$ & 0.5008 & 0.4804 & 0.0850 & 0.2113 \\
\hline$A_{2}$ & 0.3785 & 0.2133 & 0.4434 & 0.3799 \\
\hline $\mathrm{A}_{3}$ & 0.1207 & 0.3063 & 0.4716 & 0.4089 \\
\hline
\end{tabular}

That is, the actual relative weights of the 4 criteria are: $\left(\begin{array}{llll}0.1325 & 0.0890 & 0.5251 & 0.2533\end{array}\right)$. The relative importances of the 3 alternatives in terms of the first criterion are: $\left(\begin{array}{lll}0.5008 & 0.37850 .1207\end{array}\right)$, and so on.

The above values can also be viewed as the principal right eigenvectors of the following $1+4$ $=5$ perfectly consistent RCP matrices. For the case of the four criteria we have:

$$
R C P_{0}=\left[\begin{array}{llll}
1 & 1.4890 & 0.2524 & 0.5231 \\
0.6716 & 1 & 0.1695 & 0.3513 \\
3.9622 & 5.8995 & 1 & 2.0727 \\
1.9116 & 2.8462 & 0.4825 & 1
\end{array}\right]
$$

For instance, in the previous matrix the $(1,2)$ entry is equal to 1.4890 because the corresponding pairwise comparison is: $\quad 0.1325 / 0.0890(=1.4890)$. Similar interpretations hold for the remaining entries. For the three alternatives we have in terms of each of the four criteria:

$$
R C P_{1}=\left[\begin{array}{lll}
1 & 1.3230 & 4.1502 \\
0.7559 & 1 & 3.1371 \\
0.2410 & 0.3188 & 1
\end{array}\right] R C P_{2}=\left[\begin{array}{lll}
1 & 2.2552 & 1.5682 \\
0.4440 & 1 & 0.6963 \\
0.6377 & 1.4362 & 1
\end{array}\right]
$$




$$
R C P_{3}=\left[\begin{array}{lll}
1 & 0.1917 & 0.1803 \\
5.2156 & 1 & 0.9403 \\
5.5468 & 1.0635 & 1
\end{array}\right] \quad R C P_{4}=\left[\begin{array}{lll}
1 & 0.5561 & 0.5167 \\
1.7982 & 1 & 0.9291 \\
1.9355 & 1.0763 & 1
\end{array}\right]
$$

A pplying the AHP and the revised A HP it can be shown that the following results are obtained:

The AH P solution

$\begin{array}{lllll}\text { Alt. } & \text { weights } & \text { ranking } & \text { weights } & \text { ranking } \\ \mathrm{A}_{1} & 0.2073 & 3 & 0.2048 & 3 \\ \mathrm{~A}_{2} & 0.3982 & 1 & 0.3980 & 1 \\ \mathrm{~A}_{3} & 0.3945 & 2 & 0.3972 & 2\end{array}$

In reality, however, the decision maker does not know the previous real values. Instead, one has to use matrices with entries from the set: $\{1 / 9,1 / 8, \ldots, 1,2, \ldots, 8,9\}$ (that is, to use the scale presented in table 1). The CDP matrices which best approximate the previous 5 RCP matrices can be shown to be as follows. For the case of the four criteria the corresponding CDP matrix and its corresponding eigenvector is:

$$
R C P_{0} \rightarrow C D P_{0}=\left[\begin{array}{llll}
1 & 1.5000 & 0.2500 & 0.5000 \\
0.6667 & 1 & 0.1667 & 0.3333 \\
4.0000 & 6.0000 & 1 & 2.0000 \\
2.0000 & 3.0000 & 0.5000 & 1
\end{array}\right] \rightarrow\left[\begin{array}{l}
0.1304 \\
0.0870 \\
0.5217 \\
0.2609
\end{array}\right]
$$

For instance, the $(1,2)$ entry in the previous matrix is equal to 1.5000 because the corresponding entry in the RCP matrix is 1.4890 and the value 1.5000 is the closest value from the permissible values (when the scale in table 1 is used). Similar interpretations hold for the remaining entries.

Similarly with above, for the three alternatives in terms of each one of the four criteria the CDP matrices and their corresponding eigenvectors are: 


$$
\begin{aligned}
R C P_{1} \rightarrow C D P_{1} & =\left[\begin{array}{lll}
1 & 1.3333 & 4.0000 \\
0.7500 & 1 & 3.0000 \\
0.2500 & 0.3333 & 1
\end{array}\right] \rightarrow\left[\begin{array}{l}
0.5000 \\
0.3750 \\
0.1250
\end{array}\right] \\
R C P_{2} \rightarrow C D P_{2} & =\left[\begin{array}{lll}
1 & 2.2500 & 1.6000 \\
0.4444 & 1 & 0.7143 \\
0.6250 & 1.4000 & 1
\end{array}\right] \rightarrow\left[\begin{array}{l}
0.4833 \\
0.2151 \\
0.3016
\end{array}\right] \\
R C P_{3} \rightarrow C D P_{3} & =\left[\begin{array}{lll}
1 & 0.2000 & 0.1667 \\
5.0000 & 1 & 0.8889 \\
6.0000 & 1.1250 & 1
\end{array}\right] \rightarrow\left[\begin{array}{l}
0.0835 \\
0.4264 \\
0.4901
\end{array}\right] \\
R C P_{4} \rightarrow C D P_{4} & =\left[\begin{array}{lll}
1 & 0.5556 & 0.5000 \\
1.8000 & 1 & 0.8889 \\
2.0000 & 1.1250 & 1
\end{array}\right] \rightarrow\left[\begin{array}{l}
0.2083 \\
0.3734 \\
0.4183
\end{array}\right]
\end{aligned}
$$

\begin{tabular}{|c|c|c|c|c|}
\hline & & Criterio & & \\
\hline & 1 & 2 & 3 & 4 \\
\hline Alt. & (0.1304 & 0.0870 & 0.5217 & $0.2609)$ \\
\hline$A_{1}$ & 0.5000 & 0.4833 & 0.0835 & 0.2083 \\
\hline$A_{2}$ & 0.3750 & 0.2151 & 0.4264 & 0.3734 \\
\hline $\mathrm{A}_{3}$ & 0.1250 & 0.3016 & 0.4901 & 0.4183 \\
\hline
\end{tabular}

That is, the data which are assumed to be available in reality to the decision maker for the present problem are:

Similarly, as in the first part of this example, the A HP and the revised A HP yield the following results: 
The AHP solution

$\begin{array}{lllll}\text { Alt. } & \text { weights } & \text { ranking } & \text { weights } & \text { ranking } \\ \mathrm{A}_{1} & 0.2051 & 3 & 0.2049 & 3 \\ \mathrm{~A}_{2} & 0.3875 & 2 & 0.3867 & 2 \\ \mathrm{~A}_{3} & 0.4074 & 1 & 0.4084 & 1\end{array}$

These findings clearly contradict the results found in the first part of this example.

In this numerical example the weights of the two alternatives $A_{2}$ and $A_{3}$ are almost identical when the AHP is applied (namely, 0.3982 and 0.3945 , respectively) on the actual data. However, after the original Saaty scale is used, and the assumption that the decision maker is as accurate as possible is made, then the A HP yields the weights 0.3875 and 0.4074 for alternatives $A_{2}$ and $A_{3}$, respectively. In other words, the AHP causes the two alternatives to look very distinct, while in reality they are almost identical.

If for illustrative purposes one considers that the alternatives represent projects to be funded, then the A HP would suggest that project $A_{3}$ to be funded $5.14 \%$ (i.e., $(0.4074-0.3875) * 100 / 0.3875$ ) more than project $A_{2}$, while in reality project $A_{3}$ should be funded by almost $1.0 \%$ less than project $A_{2}$. That is, after the A HP or the revised AHP are applied, then it is likely that alternatives which in reality are almost identical, to appear to be rather distinct. The reverse situation is also possible to occur.

\section{COMPUTATIONAL EXPERIMENTS}

In the previous numerical example both the A HP and the revised A HP yield the same alternative ranking. However, in general this is not always the case. It is possible for the A HP to yield the correct alternative ranking and the revised AHP an incorrect one, or vice-versa. In order to study the performance of the two methods in depth, a simulation approach was followed. Random problems with different number of criteria and alternatives were generated and then treated as in the previous example.

For each such random problem actual relative weights were assumed as in the previous numerical example. However, because the Saaty matrices use values from the set: $\{1 / 9,1 / 8, \ldots, 1,2, \ldots, 8,9\}$ only the random problems which are associated with RCP matrices with entries within the continuous interval 
$[1 / 9,9 / 1]$ were considered. Each test problem was treated as in the previous example. If the AHP or the revised A HP yielded a ranking different than the one derived when actual data were used, then the case was recorded as a failure. Tables 2 and 3 present the results of this simulation approach for random problems of different sizes. Table 2 deals with the AHP and table 3 with the revised AHP. Figures 1 and 2 graphically depict the results presented in tables 2 and 3, respectively. The simulation program was written in FORTRAN and the random numbers were generated by using the IM SL library of subroutines.

The findings reveal that the number of alternatives in a decision problem is very critical. As the number of alternatives increases, so does the failure rate of the AHP and the revised AHP. This was expected since in [11] it was found that the number of members in a fuzzy set dramatically influences the performance of the eigenvalue approach in deriving accurate member ratings. However, in the present investigation the failure rates are much higher than the ones reported in [11]. It is worthy to note here that in the tests reported in [11] the revised A HP performed significantly better than the original A HP. The same findings also demonstrate that the number of criteria seems to be without any particular influence on the final results.

It should be noted here that in these simulation experiments it was assumed that the decision maker is as accurate as possible. This assumption is imposed by the introduction of the concept of the CDP matrices (described in an earlier section). Clearly, this is an assumption which works in favor of the two methods. If one accepts the premise that the decision maker cannot always be that effective in his/her judgments, then it follows that in real life applications the failures of the two methods occur even more frequently and intensively than what is reported in this paper. 
Table 2. Failure rates are based on 1,000 randomly generated problems

(the A HP case)

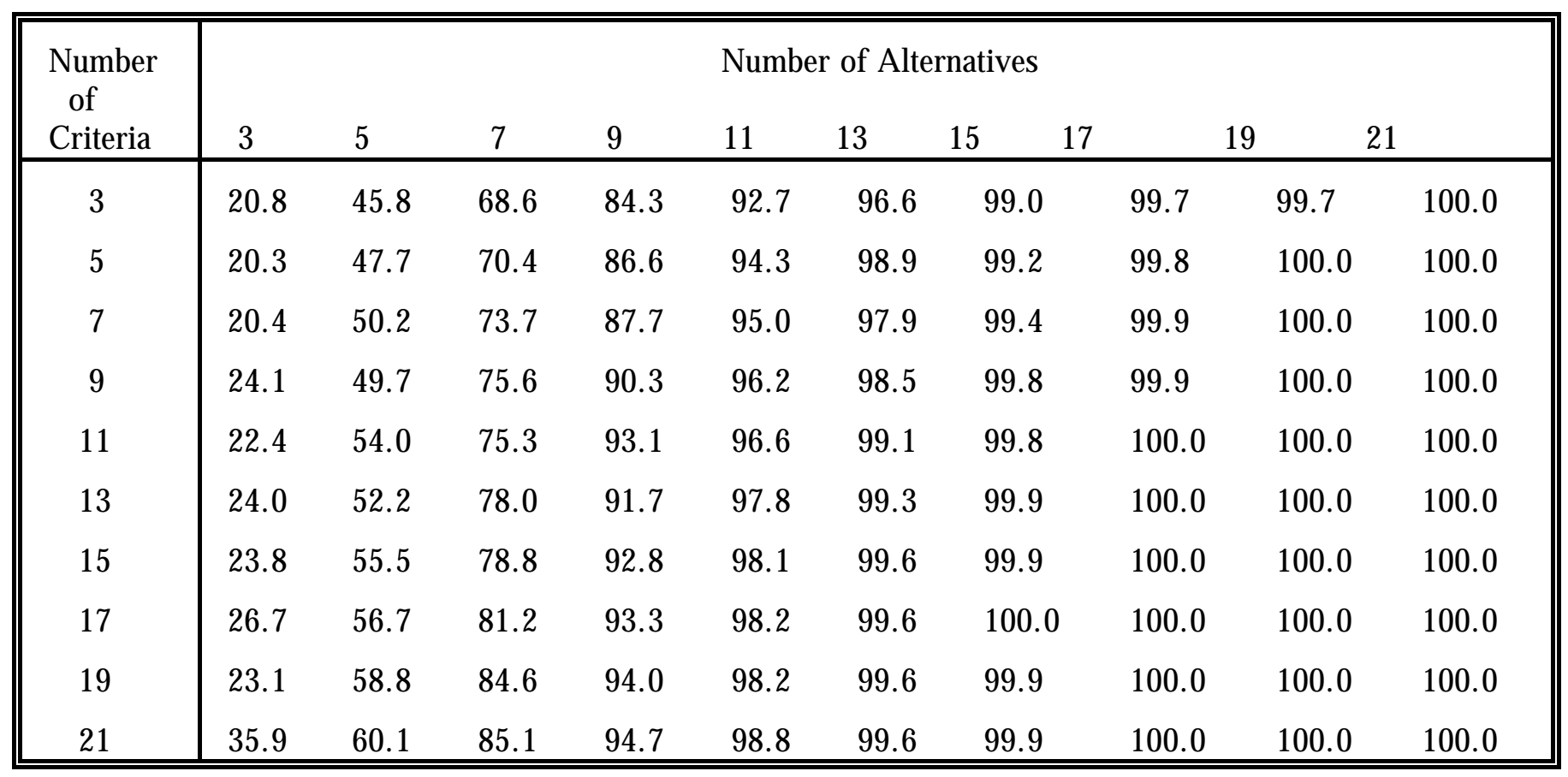

Table 3. Failure rates are based on 1,000 randomly generated problems

(the revised AHP case)

\begin{tabular}{||c|ccccccccccc||}
\hline \hline $\begin{array}{c}\text { Number } \\
\text { of } \\
\text { Criteria }\end{array}$ & 3 & 5 & 7 & 9 & 11 & 13 & 15 & 17 & 19 & 21 \\
\hline 3 & 22.8 & 48.1 & 69.3 & 86.0 & 93.4 & 97.5 & 99.3 & 99.6 & 99.8 & 100.0 \\
5 & 20.4 & 46.8 & 72.6 & 87.5 & 93.5 & 98.4 & 99.5 & 99.8 & 99.9 & 100.0 \\
7 & 21.1 & 51.4 & 75.3 & 88.5 & 95.7 & 99.1 & 99.5 & 100.0 & 100.0 & 100.0 \\
9 & 24.1 & 52.4 & 75.0 & 89.7 & 97.4 & 99.0 & 100.0 & 99.8 & 100.0 & 100.0 \\
11 & 23.6 & 56.1 & 76.6 & 92.8 & 96.0 & 98.9 & 99.7 & 100.0 & 100.0 & 100.0 \\
13 & 25.7 & 55.2 & 79.3 & 92.6 & 98.0 & 99.2 & 100.0 & 100.0 & 100.0 & 100.0 \\
15 & 26.9 & 55.9 & 82.1 & 94.3 & 97.7 & 99.5 & 100.0 & 99.9 & 100.0 & 100.0 \\
17 & 27.7 & 57.0 & 82.7 & 93.9 & 98.7 & 99.3 & 100.0 & 100.0 & 100.0 & 100.0 \\
19 & 25.5 & 56.6 & 84.0 & 94.0 & 98.7 & 99.7 & 99.9 & 100.0 & 100.0 & 100.0 \\
21 & 28.2 & 59.8 & 83.1 & 93.6 & 98.5 & 99.7 & 100.0 & 100.0 & 100.0 & 100.0 \\
\hline \hline
\end{tabular}






Fig. 1. Failure rates are based on 1,000 randomly generated problems (the A HP case)



Fig. 2. Failure rates are based on 1,000 randomly generated problems (the revised A HP case) 
From the above observations it is concluded that if one uses the A HP or the revised A HP in order to select the best alternative, then he/she may instead select an alternative which is not the best. Similarly, if one is interested in determining the relative weights of the alternatives, then distortions in the calculated weights are possible.

The answer to the critical question whether the numerical anomalies reported here are severe or not, depends on the particular application under consideration, and no generalizations can be made. If the impact of choosing a nonoptimal al ternative is not that severe, then the decision maker should not be highly concerned. H owever, if the application is very critical, then the present investigation may serve as a warning for caution before reaching the final decision. The present investigation reaffirms the belief of many practitioners today, that multi-attribute decision-making methods should be used only as decision support tools, and not to be taken literally.

\section{CONCLUDING REMARKS}

In this paper we investigated the reversals in the al ternative ranking which occur when Saaty pairwise comparison matrices are used as input in decision-making problems. Saaty pairwise comparison matrices are widely considered as successful means for gathering data in many real world decision-making situations. The AHP and some of its variants have found a considerable appeal in approaching many real life engineering applications. M any computer packages have been developed (see, for instance, [13]) to automate their application to real life problems.

The forward error analysis in this paper reveals that when the A HP or the revised AHP methods are used in combination with the eigenvalue approach for processing the input data, then severe alternative ranking failures are possible. These failures are more dramatic as the number of al ternatives increases. How ever, even for decision problems with a few alternatives the possibility of ranking alterations is significantly high. The number of criteria or the decision-making method used (i.e., the A HP or the revised A HP) seem to be without any particular influence in the above rates. Therefore, these results can be used to properly guide the decision maker when applying these methods to real life engineering problems.

The present paper provides a justification on why the application of the AHP (or the revised AHP) should be treated with some skepticism and their results not to be taken literally. This consideration comes in symphony with the concerns expressed by other researchers in the past (see, for instance, [24], [29], [25], [26], [30], and [33]). However, 
the assumption that the decision maker is as accurate as possible in deriving his/her judgments (i.e., the concept of the CDP matrices ) and its application in evaluating multi-criteria decision making methods is a very new one.

It is worthy to emphasize here that in under less than perfect conditions the real failure rates can be even more

dramatic. It should also be kept in mind, that the present investigation is based on the assumption that the actual data for evaluating alternatives in terms of decision criteria, take on continuous values. Therefore, the previous results are subject to this continuity assumption.

The present results, along with the realization that data in multi-attribute decision-making problems are constant, urge for the need to perform a sensitivity analysis on any real life decision problem. M asuda in [41] presents a very interesting approach for performing a sensitivity analysis when the AHP is used. A nother approach for performing a systematic sensitivity analysis when the A HP is used, is due to Sanchez and Triantaphyllou [42]. N ote that the computer software EXPERT CHOICE, which is based on the AHP, also allows for some preliminary sensitivity analysis. It is highly advised that a sensitivity analysis should be always be applied in real applications, especially when some alternatives appear to be close to each other.

Finally, it should be stated here that the same investigation, besides being a mechanism for testing future models, also reveals that there is an open problem in this class of decision-making models and more research in this topic is needed.

\section{REFERENCES}

1. T.L. Saaty. A Scaling M ethod for Priorities in Hierarchical Structures. J ournal of Mathematical Psychology, 15, 57-68 (1977).

2. T.L. Saaty. Exploring the interface between hierarchies, multiple objects and fuzzy sets, J ournal of Fuzzy Sets and Systems, 1, 57-68 (1978).

3. T.L. Saaty. The Analytic Hierarchy Process, M cGraw-Hill International, New Y ork, NY (1980).

4. A.T.W. Chu, R.E. Kalaba, and K. Spingarn. A Comparison of Two M ethods for Determining the W eights of Belonging to Fuzzy Sets. J ournal of Optimization Theory and Applications, 27, 531-538 (1979). 
5. V.V. Federov, V.B. Kuz' min, V.B., and A.I. V ereskov. M embership Degrees D etermination from Saaty M atrix Totalities, Institute for System Studies, M oscow, U SSR. Paper appeared in: Approximate Reasoning in Decision Analysis, M.M. Gupta, and E. Sanchez (editors), North-Holland Publishing Company, 23-30 (1982).

6. J.I. Khurgin, and V.V. Polyakov. Fuzzy A nalysis of the Group Concordance of Expert Preferences, defined by Saaty Matrices. Fuzzy Sets Applications, Methodological Approaches and Results, Akademie-Verlag Berlin, 111-115 (1986).

7. J.I. Khurgin, and V.V. Polyakov (1986). Fuzzy A pproach to the A nalysis of Expert Data. Fuzzy Sets Applications, Methodological Approaches and Results, A kademie-Verlag Berlin, 116-124 (1986).

8. E. Triantaphyllou and S.H. M ann. An Examination of the Effectiveness of M ulti-Dimensional Decision-M aking M ethods: A Decision-M aking Paradox, International J ournal of D ecision Support Systems, 5, 303-312 (1989).

9. E. Triantaphyllou, P.M. Pardalos, and S.H. M ann. A M inimization A pproach to M embership Evaluation in Fuzzy Sets and Error A nalysis, J ournal of Optimization Theory and Applications, 66(2), 275-287 (1990).

10. E. Triantaphyllou, P.M. Pardalos, and S.H. M ann. The Problem of Evaluating M embership V alues in Real W orld Situations. O perations Research and Artificial Intelligence: The Integration of P roblem Solving Strategies, (D.E. Brown and C.C. White III, editors), Kluwer Academic Publishers, 197-214 (1990).

11. E. Triantaphyllou and S.H. M ann. A n Evaluation of the Eigenvalue A pproach for D etermining the M embership V alues in Fuzzy Sets. Fuzzy Sets and Systems. 35(3), 295-301 (1990).

12. L.G. Vargas. Reciprocal M atrices with Random Coefficients. Mathematical Modeling, 3, 69-81 (1982).

13. Decision Support Software, Inc. Expert Choice Software Package. M cL ean, Virginia (1986).

14. R. Putrus. Accounting for Intangibles in Integrated M anufacturing (nonfinancial justification based on the A nalytical Hierarchy Process). Information Strategy, 6, 25-30 (1990).

15. C. Falkner and S. Benhajla. Multi-attribute Decision Models in the Justification of CIM Systems. The Engineering Economist, 35, 91-114 (1990).

16. K. Swann and W.D. O'Keefe. Advanced Manufacturing Technology: Investment Decision Process. Management Decisions, 28(1), 20-31 (1990). 
17. G.C. Roper-Lowe and J.A. Sharp. The A nalytic Hierarchy Process and its Application to an Information Technology Decision. Operational Research Society Ltd., 41(1), 49-59 (1990).

18. J. Finnie. The Role of Financial A ppraisal in Decisions to Acquire Advanced M anufacturing Technology. Accounting and Business Research, 18(7), 133-139 (1988).

19. T. O. Boucher and E.L. M cStravic. M ulti-attribute Evaluation within a Present V alue F ramework and its Relation to the A nalytic Hierarchy Process. The Engineering Economist, 37(1), 55-71 (1991).

20. R.N. Wabalickis. Justification of FM S with the A nalytic Hierarchy Process. J ournal of M anufacturing Systems, 17(3), $175-182$ (1988).

21. K.E. Cambron and G.W. Evans. Layout Design U sing the A nalytic Hierarchy Process. Computers \& IE. 20(2) 221-229 (1991).

22. L. Wang and T. Raz. A nalytic Hierarchy Process Based on Data Flow Problem. Computers \& IE. 20(3) 355365 (1991).

23. A. A rbel and A. Seidmann. Selecting a M icrocomputer for Process Control and D ata Acquisition. IEE Trans. 16(1) (1984).

24. V. Belton and T. Gear. On a Short-coming of Saaty's M ethod of A nalytic Hierarchies. O mega, 228-230 (1983).

25. J.S. Dyer and H.V. Ravinder. Irrelevant A Iternatives and the A nalytic Hierarchy Process. Working Paper, Department of M anagement, The U niversity of Texas at A ustin, (1983).

26. J.S. Dyer and R.E. Wendell. A Critique of the Analytic Hierarchy Process. Working Paper 84/85-4-24, D epartment of M anagement, The U niversity of Texas at A ustin, (1985).

27. T.L. Saaty. A xiomatic F oundations of the A nalytic Hierarchy Process. Management Science, 32(7), 841-855 (1983).

28. T.L. Saaty. Rank Generation, Preservation, and Reversal in the A nalytic Hierarchy Process. Decision Sciences, 18, $157-177$ (1987).

29. J.S. Dyer. Remarks on the A nalytic Hierarchy Process. Management Science, 36(3), 249-258 (1990).

30. J.S. Dyer. A Clarification of "Remarks on the A nalytic Hierarchy Process". Management Science, 36(3), 
274-275 (1990).

31. T.L. Saaty. An Exposition of the AHP in Reply to the Paper "Remarks on the A nalytic Hierarchy Process". M anagement Science, 36(3), 259-268 (1990).

32. P.T. Harker and L.G. Vargas. Reply to "Remarks on the A nalytic Hierarchy Process". M anagement Science, 36(3), 269-273 (1990).

33. R.L. Winkler. Decision M odeling and Rational Choice: A HP and Utility Theory. Management Science, 36(3), $247-248$ (1990).

34. D.E. Bell and P.H. Farquhar. Perspectives on Utility Theory. Operations Research, 34, 179-183 (1986).

35. R.M. Hogarth and M.W. Reder, (Eds.). Rational Choice: The Contrast Between Economics and Psychology, University of Chicago Press, Chicago, Illinois, (1986).

36. M. Weber and C. Camerer. Recent Developments in Modeling Preferences Under Risk. OR Spectrum, 9, 129-151 (1987).

37. B.R. M unier (Ed.). Risk, Decision and Rationality. D. Reidel, Dordrecht, Holland, 1988.

38. P.C. Fishburn and I.H. LaV alle (Eds.). Annals of O perations Research. Volume 19: Choice U nder U ncertainty. J.C. Baltzer, Basel, Switzerland, 1989.

39. R.K. Sarin. A nalytical Issues in Decision M ethodology. In I. Horowitz (Ed. ), Organization and D ecision Theory, Kluwer- Nijhoff, Dordrecht, Holland, 156-172 (1989).

40. E. Triantaphyllou, F.A. Lootsma, P.M. Pardalos, and S.H. M ann. On the Evaluation and A pplication of Different Scales For Quantifying Pairwise Comparisons in Fuzzy Sets. Multi-Criteria Decision Analysis, 3(3), 133-155 (1993).

41. T. M asuda. Hierarchical Sensitivity A nalysis of the Priorities U sed in the A nalytic Hierarchy Process. Int. J. of Systems Science, 21(2), 415-427 (1990).

42. E. Triantaphyllou and A. Sanchez and E. Triantaphyllou. A Sensitivity A nalysis Approach for Some Deterministic Multi-Criteria Decision-M aking. Decision Sciences, 28(1), 151-194 (1997). 